\title{
(2) OPEN ACCESS \\ Prehospital clinical signs are a poor predictor of raised intracranial pressure following traumatic brain injury
}

\author{
Ewoud ter Avest 이 , ${ }^{1,2}$ Sam Taylor, ${ }^{1}$ Mark Wilson, ${ }^{1,3}$ Richard L Lyon ${ }^{1,4}$
}

\begin{abstract}
Handling editor Caroline Leech

${ }^{1}$ Air Ambulance Kent Surrey and Sussex, Redhill, UK ${ }^{2}$ Universitair Medisch Centrum Groningen, Department of Emergency Medicine, University of Groningen, Groningen, The Netherlands

${ }^{3}$ Neurotrauma Centre, Imperial College London, London, UK ${ }^{4}$ School of Health Sciences, University of Surrey, Guildford, UK
\end{abstract}

\section{Correspondence to} Dr Ewoud ter Avest, Air Ambulance Kent, Surrey and Sussex, Redhill, Surrey RH1 5YP, UK;

Ewoudteravest@aakss.org.uk

Received 17 March 2020 Revised 16 August 2020

Accepted 23 August 2020 Published Online First 18 September 2020

\section{Check for updates}

(C) Author(s) (or their employer(s)) 2021. Re-use permitted under CC BY-NC. No commercial re-use. See rights and permissions. Published by BMJ.

To cite: ter Avest E, Taylor S, Wilson M, et al. Emerg Med J 2021;38:21-26.

\begin{abstract}
Background For the prehospital diagnosis of raised intracranial pressure (ICP), clinicians are reliant on clinical signs such as the Glasgow Coma Score (GCS), pupillary response and/or Cushing's triad (hypertension, bradycardia and an irregular breathing pattern). This study aimed to explore the diagnostic accuracy of these signs as indicators of a raised ICP.

Methods We performed a retrospective cohort study of adult patients attended by a Helicopter Emergency Medical Service (Air Ambulance Kent, Surrey Sussex), who had sustained a traumatic brain injury (TBI), requiring prehospital anaesthesia between 1 January 2016 and 1 January 2018. We established optimal cut-off values for clinical signs to identify patients with a raised ICP and investigated diagnostic accuracy for combinations of these values.
\end{abstract}

Results Outcome data for 249 patients with TBI were available, of which 87 (35\%) had a raised ICP. Optimal cut-off points for systolic blood pressure (SBP), heart rate (HR) and pupil diameter to discriminate patients with a raised ICP were, respectively, $>160 \mathrm{~mm} \mathrm{Hg},<60 \mathrm{bpm}$ and $>5 \mathrm{~mm}$. Cushing criteria (SBP >160 mm Hg and $\mathrm{HR}<60 \mathrm{bpm}$ ) and pupillary response and size were complimentary in their ability to detect patients with a raised ICP. The presence of a fixed blown pupil or a Cushing's response had a specificity of 93.2 (88.2$96.6) \%$, and a positive likelihood ratio (LR+) of 5.4 (2.9-10.2), whereas sensitivity and LR- were only 36.8 $(26.7-47.8) \%$ and $0.7(0.6-0.8)$, respectively, (Area Under the Curve (AUC) 0.65 (0.57-0.73)). Sensitivity analysis revealed that optimal cut-off values and resultant accuracy were dependent on injury pattern. Conclusion Traditional clinical signs of raised ICP may under triage patients to prehospital treatment with hyperosmolar drugs. Further research should identify more accurate clinical signs or alternative non-invasive diagnostic aids in the prehospital environment.

\section{INTRODUCTION}

Many patients attended by prehospital critical care teams suffer from a traumatic brain injury (TBI). ${ }^{1}$ The mainstay of treatment in these patients is to prevent secondary brain injury by minimising the ischaemic penumbra by measures to maintain cerebral blood flow (CBF) and cerebral oxygenation.

Early recognition and treatment of increased intracranial pressure (ICP) is one of the elements of prehospital treatment in these patients. As patients with TBI have a disturbed cerebral autoregulation, a reduced cerebral perfusion pressure (CPP) due to

\section{Key messages}

What is already known on this subject

- Prehospital clinicians are reliant on clinical signs for detection of a raised intracranial pressure (ICP) in patients with traumatic brain injury (TBI) to guide temporising ICP-lowering treatment with hyperosmolar drugs.

- Clinical criteria for the detection of a raised ICP are not well defined: blood pressure and heart rate (HR) cut-off values to define Cushing's response do not exist, and a uniform definition of 'fixed dilated pupils' in the context of TBI is lacking.

- Until now, it is not clear what the diagnostic accuracy of clinical criteria for detection of a raised ICP is.

\section{What this study adds}

- This is a retrospective cohort study of all adult patients with TBI requiring prehospital anaesthesia who were attended by the Air Ambulance Kent, Surrey \& Sussex.

- We found that even when optimal cut-off points for systolic blood pressure, HR and pupil diameter are used, clinical criteria have a low sensitivity for detecting a raised ICP in patients with TBI in the prehospital setting.

- Further research should identify more accurate clinical signs or alternative non-invasive diagnostic aids in the prehospital environment.

an elevated ICP is not compensated by an increase in $\mathrm{CBF}$, and may even result in a reduction of $\mathrm{CBF}^{23}$ Therefore, it is important to detect ICP rises early.

Prehospital clinicians rely largely on clinical signs to identify patients with a raised ICP. These clinical signs, including a reduction in Glasgow Coma Score (GCS), fixed dilated pupil(s) and/or Cushing's triad (hypertension, bradycardia and an irregular breathing pattern), are the consequence of intracranial changes due to a rise in ICP. ${ }^{4}$ In many (prehospital) treatment guidelines, they are used to guide temporising ICP-lowering treatment with hyperosmolar drugs as hypertonic saline (HS) or mannitol, next to other standard ICP-lowering measures, such as head-up transport, adequate sedation and analgesia, muscle paralysis and neuroprotective ventilation. ${ }^{5-8}$

In-hospital, using clinical signs in combination with radiological imaging, to guide treatment 
of raised ICP has been shown to be equivalent to direct ICP measurements in terms of impact on patient outcome. ${ }^{9}$ However, in the prehospital situation, there are several potential limitations. First, radiological imaging modalities are not available. Second, clinical criteria for the detection of a raised ICP were not well defined: blood pressure (BP) and heart rate cut-off values to define Cushing's response do not exist. In addition, a uniform definition of 'fixed dilated pupils' in the context of TBI is lacking. ${ }^{7}$ Finally, the Cushing's response may be obscured by significant comorbidity, especially (haemorrhagic) shock. This may impact the diagnostic accuracy of clinical criteria to detect a raised ICP.

In this study, we aimed to establish evidence-based cut-off values for clinical signs to identify patients with a raised ICP and investigated diagnostic accuracy for (combinations of) these variables.

\section{METHODS}

\section{Study setting and design}

This is a retrospective cohort study of all adult patients with TBI requiring prehospital anaesthesia who were attended by the Air Ambulance Kent, Surrey \& Sussex (AAKSS) between 1 January 2016 and 1 January 2018. AAKSS is a Helicopter Emergency Medical Service (HEMS) covering three counties in the south east of England with a resident population of 4.5 million and transient population of up to 8 million. Two doctor/paramedic teams respond in either a helicopter or response car from one base. The service attends approximately 2000 patients per year.

\section{Study population}

Patients were eligible for inclusion in the study if they were 18 years or older at the time of injury and had sustained blunt head trauma (with or without other traumatic injury) requiring prehospital emergency anaesthesia. Excluded patients included those with non-traumatic intracranial pathology resulting in a reduced GCS (including intracranial bleeds and space occupying lesions with or without signs of raised ICP on CT); patients who died before arrival in hospital and patients for whom follow-up data regarding ICP raise were unavailable.

\section{Prehospital treatment}

All patients attended by AAKSS with TBI receive neuroprotective care. This includes RSI with opiate premedication, ${ }^{10}$ neuroprotective ventilation (aiming for normocapnia and normal oxygen saturations $\left.\left(\mathrm{SpO}_{2}\right)\right)$, head up transport where possible and transport without a cervical collar. AAKSS crews carry $500 \mathrm{~mL}$ of HS ( $\mathrm{NaCl} 5 \%$ ). Patients receive $6 \mathrm{~mL} / \mathrm{kg}$ to a maximum of $350 \mathrm{~mL}$ as per service operating procedure (SOP), when they show 'signs of actual or impeding cerebral herniation as a result of severe head injury as evidenced by unilateral or bilateral pupil dilatation in association with GCS $<8$ or progressive hypertension (systolic blood pressure (SBP) $160 \mathrm{~mm} \mathrm{Hg}$ ) and bradycardia (<60 bpm)'. HS was not administered by other prehospital care providers in the AAKSS operating region.

\section{Clinical endpoints}

The endpoints of interest in this study were defined as:

- Optimal cut-off values for clinical variables to identify patients with an increased ICP as a result of TBI in the prehospital setting.

- The accuracy of the best combination of clinical criteria for the identification of patients with a raised ICP as a result of TBI.

\section{Definitions}

As direct in-hospital ICP measurements were not performed in the majority of patients, a surrogate combined endpoint was used to establish which patients had an elevated ICP as a result of their TBI. ICP was regarded as elevated when, in the first 24 hours after hospital admission, either ICP was $>25 \mathrm{~mm} \mathrm{Hg}$ (as measured invasively) $^{2}$ or when patients underwent craniectomy within 24 hours of admission. When neither craniectomy nor direct ICP measurement was performed, reported CT scan results were used as a surrogate for ICP measurements. ICP was presumed to be elevated when the radiology report mentioned one of the following: herniation (subfalcine, transtentorial, uncal or cerebellar (tonsillar)), midline shift or significant mass effect with either subdural haematoma, epidural haematoma, cerebral contusion or traumatic subarachnoid bleed. ${ }^{11}$ When any of these injuries were present without a mass effect as described by the radiologist, ICP was regarded as not elevated. For the purpose of this study, Cushing's response was defined as the presence of hypertension and bradycardia. No attempt was made to assess irregular respiration as this was a retrospective study relying on documentation. Code red patients were defined as patients with suspected ongoing bleeding who, according to the treating clinicians, had a potential to require prehospital or in-hospital transfusion of blood products.

\section{Data acquisition}

Data on patient characteristics, prehospital vital signs and treatments were retrieved from the electronic patient clinical record system AAKSS uses (HEMSBase V.2.0, Medic One Systems, UK). The following data were retrieved from the patient record: patient characteristics (age and gender), clinical findings on arrival of HEMS (first recorded heart rate (HR), SBP, diastolic blood pressure (DBP), mean arterial pressure (MAP), GCS, including eye-motor and verbal subscores), pupil diameters and responsiveness, body regions injured (isolated head injury or multisystem injuries), highest SBP, DBP and MAP and lowest HR anytime during prehospital care. Administration of HS, CT scan results, ICP measurements and surgical notes from the first 24 hours in hospital were noted. To minimise the impact of spurious readings for HR and BP, highest and lowest values were calculated as averages of three consecutive recordings.

\section{Patient and public involvement}

Lay representation on the AAKSS charity board expressed support for further research being undertaken into TBI. Patients were not directly involved in the design, recruitment or conduct of the study. The results and impact of the study will be shared with the lay representatives.

\section{Statistics}

Descriptive statistics are given as mean $(95 \% \mathrm{CI})$ or median (IQR). Missing data are reported in the 'Results' section according to the Standards for Reporting Diagnostic Accuracy (STARD) guideline. Comparisons across groups were made using Student's t-test, Mann-Whitney U test or Fisher's exact test where appropriate. Optimal cut-off values to discriminate between subjects with and without an increased ICP were subsequently determined for the continuous variables $\mathrm{HR}$ and SBP under the condition of equal 'cost' of misclassification of cases and non-cases by calculating the maximum Youden Index for each variable. Likelihood ratios, sensitivities and specificities were calculated for (combinations of) clinical criteria to detect an increased ICP. Receiver Operating Characteristics 


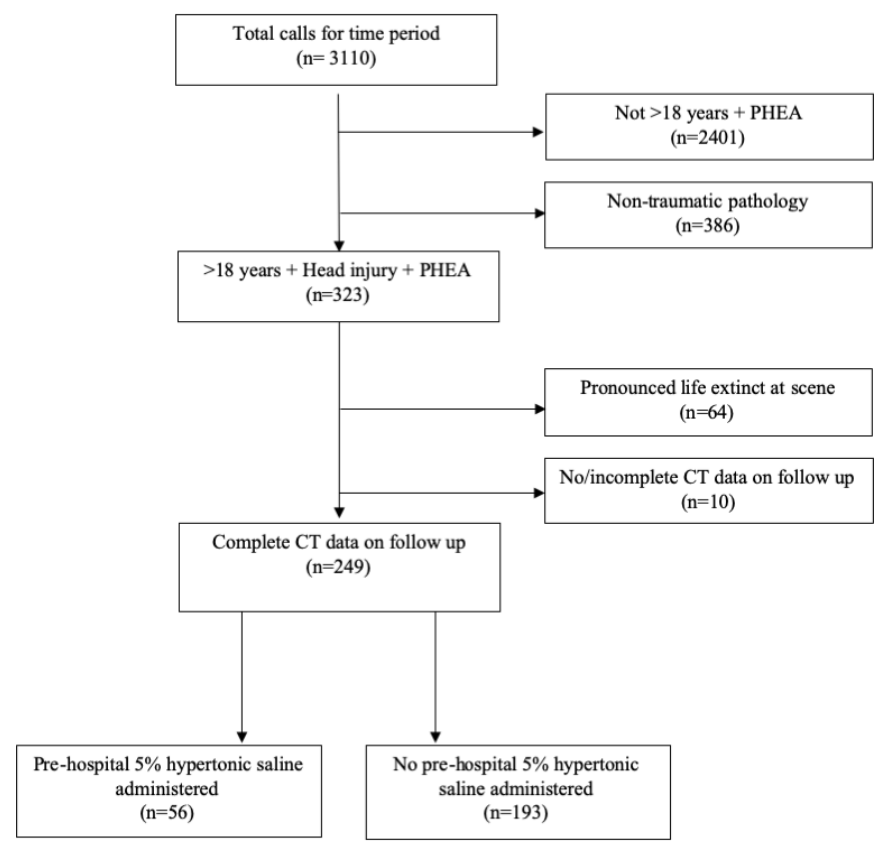

Figure 1 Study population. PHEA, prehospital emergency anaesthesia.
(ROC) analysis was performed to calculate and compare Areas under the Curve (AUCs) for different (combinations) of clinical criteria. Preplanned sensitivity analysis was performed for subgroups of patients with isolated head injuries and for patients who were declared code red. A p value $<0.05$ was considered statistically significant. Statistical analysis was performed using SPSS V.26 for Mac statistical package (SPSS, Chicago, Illinois, USA).

\section{RESULTS}

\section{Study population}

During the study period, a total of 3100 patients were attended by AAKSS. Of which, 323 patients fulfilled inclusion criteria, and for 249 of them clinical criteria and follow-up data on (surrogate) ICP were available (figure 1). All of these patients had recordable HR and BP.

One hundred and sixty-two patients $(65.0 \%)$ had no raised ICP, whereas eighty-seven (35.0\%) had either a raised ICP $(n=10)$, underwent craniectomy within 24 hours $(n=2)$ or had at least one CT surrogate for raised ICP $(n=75): 6$ patients demonstrated herniation, 49 midline shift and 20 a significant mass effect. These patients are further referred to as patients with a raised ICP. The characteristics of our study population stratified by their ICP are presented in table 1 .

\begin{tabular}{|c|c|c|c|c|}
\hline & $\begin{array}{l}\text { All subjects } \\
(\mathrm{n}=249)\end{array}$ & $\begin{array}{l}\text { Normal ICP } \\
(n=162)\end{array}$ & $\begin{array}{l}\text { Raised ICP } \\
(n=87)\end{array}$ & $P$ value* \\
\hline \multicolumn{5}{|l|}{ Patient characteristics } \\
\hline Age (year) & $49(46-51)$ & $47(43-50)$ & $54(49-59)$ & 0.009 \\
\hline Gender (male), $\mathrm{n}(\%)$ & $183(73.4)$ & $117(72.2)$ & $66(75.8)$ & 0.36 \\
\hline \multicolumn{5}{|l|}{ Injuries } \\
\hline Isolated TBI, n (\%) & $93(37.3)$ & $46(28.3)$ & $47(54.0)$ & $<0.0001$ \\
\hline Multisystem injuries, $\mathrm{n}(\%)$ & $154(61.8)$ & $114(70.4)$ & $40(46.0)$ & $<0.001$ \\
\hline Code red, n (\%) & $53(21.3)$ & $40(24.7)$ & $13(14.9)$ & 0.07 \\
\hline Missing & $2(0.8)$ & $2(1.2)$ & & \\
\hline \multicolumn{5}{|l|}{ Vital signs on arrival HEMS } \\
\hline GCS before intubation & $7(7)$ & $6(6)$ & $5(5)$ & $<0.001$ \\
\hline Eyes score & $1(2)$ & $2(2)$ & $1(1)$ & 0.001 \\
\hline Motor score & $4(3)$ & $5(2)$ & $2(4)$ & $<0.001$ \\
\hline Verbal score & $2(2)$ & $2(3)$ & $1(1)$ & 0.001 \\
\hline First SBP recorded & $144(132-157)$ & $131(120-141)$ & $168(140-196)$ & 0.005 \\
\hline First HR recorded & $106(93-118)$ & $102(91-113)$ & $112(83-141)$ & 0.47 \\
\hline \multicolumn{5}{|l|}{ Vital signs anytime during HEMS treatment } \\
\hline Highest prehospital SBP† & $153(149-157)$ & $149(145-153)$ & $160(154-167)$ & 0.003 \\
\hline Highest prehospital DBP† & $103(100-106)$ & $102(98-106)$ & $104(99-110)$ & 0.48 \\
\hline Highest prehospital MAP† & $120(116-123)$ & $114(113-122)$ & $123(118-129)$ & 0.11 \\
\hline Lowest prehospital HR† & $82(79-85)$ & $85(82-89)$ & $77(72-77)$ & 0.003 \\
\hline $\mathrm{SBP}>160 \mathrm{~mm} \mathrm{Hg}, \mathrm{n}(\%)$ & $94(36.3)$ & $51(31.5)$ & $43(49.5)$ & 0.004 \\
\hline $\mathrm{HR}<60 \mathrm{bpm}, \mathrm{n}(\%)$ & $41(15.8)$ & $19(11.7)$ & $22(25.3)$ & 0.007 \\
\hline $\mathrm{SBP}>160 \mathrm{~mm} \mathrm{Hg}$ and $\mathrm{HR}<60 \mathrm{bpm}, \mathrm{n}(\%)$ & $15(6.0)$ & $5(3.0)$ & $10(11.4)$ & 0.011 \\
\hline Fixed dilated pupil(s), $n$ (\%) & $31(12.4)$ & $6(3.7)$ & $25(28.7)$ & $<0.001$ \\
\hline \multicolumn{5}{|l|}{ HEMS hyperosmolar therapy } \\
\hline HS 5\%, n (\%) & $58(23.3)$ & $15(9.3)$ & $43(49 / 4)$ & $<0.001$ \\
\hline
\end{tabular}

Represented are mean values except for GCS (median (IQR)).

* Represented are unadjusted $\mathrm{p}$ values.

†To minimise the impact of spurious readings, highest/ lowest values were averages of three consecutive recordings: the highest/lowest recorded, and the readings before and after.

DBP, diastolic blood pressure; GCS, Glasgow Coma Scale; HEMS, Helicopter Emergency Medical Service; HR, heart rate; HS, hypertonic saline; ICP, intracranial pressure; MAP, mean arterial pressure; SBP, systolic blood pressure; TBI, Traumatic Brain Injury. 
Table 2 Diagnostic accuracy of (individual components of) clinical criteria to discriminate the presence of a raised intracranial pressure in patients with severe blunt head injury $(n=249)$

\begin{tabular}{|c|c|c|c|c|c|}
\hline & Sensitivity & Specificity & $\mathrm{LR}+$ & LR- & AUC \\
\hline Fixed pupil(s) $>5 \mathrm{~mm}$ & $28.7(19.5-39.4)$ & $96.3(92.1-98.6)$ & $7.8(3.3-18.2)$ & $0.7(0.7-0.9)$ & $0.63(0.55-0.70)$ \\
\hline $\mathrm{HR}<60 \mathrm{bpm}$ & $25.3(16.6-35.8)$ & $88.3(82.3-92.8)$ & $2.2(1.2-3.8)$ & $0.9(0.7-1.0)$ & $0.57(0.49-0.64)$ \\
\hline $\mathrm{SBP}>160 \mathrm{~mm} \mathrm{Hg}$ & $49.4(38.5-60.4)$ & $68.5(60.8-75.6)$ & $1.6(1.2-2.1)$ & $0.7(0.6-0.9)$ & $0.59(0.52-0.67)$ \\
\hline $\mathrm{HR}<60$ and $\mathrm{SBP}>160$ & $11.5(5.7-20.1)$ & $96.9(92.9-98.9)$ & $3.7(1.3-10.6)$ & $0.9(0.8-1.0)$ & $0.54(0.47-0.62)$ \\
\hline Fixed pupils $>5 \mathrm{~mm}$ or $(\mathrm{HR}<60$ and $\mathrm{SBP}>160)$ & $36.8(26.7-47.8)$ & $93.2(88.2-96.6)$ & $5.4(2.9-10.2)$ & $0.7(0.6-0.8)$ & $0.65(0.57-0.73)$ \\
\hline
\end{tabular}

AUC, Area Under the Curve; HR, heart rate; LR+, positive likelihood ratio; LR-, negative likelihood ratio; SBP, systolic blood pressure.

Patients who had raised ICP more often suffered from multisystem injuries (as opposed to isolated TBI), were on average older, had a higher SBP and a lower HR on arrival of the HEMS team, and often had one or two dilated and unreactive pupils compared with patients who did not have a raised ICP.

\section{Accuracy of clinical criteria for the detection of a raised ICP in the prehospital environment}

AUCs for SBP, HR and pupil size were respectively 0.66 (0.59$0.73), 0.43(0.35-0.51)$ and $0.63(0.55-0.70)$. Optimal cut-off points (as derived using ROC statistics) for SBP, HR and pupil diameter to discriminate patients who had a raised ICP were respectively $>160 \mathrm{~mm} \mathrm{Hg},<60 \mathrm{bpm}$ and $>5 \mathrm{~mm}$. Diagnostic accuracy of different clinical criteria and combinations of criteria for the detection of a raised ICP are shown in table 2 . The presence of one or two fixed dilated pupils was the best overall predictor of a raised ICP with an AUC of 0.63. Whereas a Cushing's response had a high specificity $(96.9 \%)$ for the detection of a raised ICP, sensitivity was very low (11.5\%). As expected, pupil changes and Cushing criteria were complimentary in their ability to detect a raised ICP: 7 patients with a raised ICP had no fixed dilated pupil but met Cushing's criteria, whereas 22 patients did not fulfil Cushing's criteria, but did have 1 or 2 fixed dilated pupils. Both criteria combined (presence of (a) fixed dilated pupil(s) $>5 \mathrm{~mm}$ or a Cushing's response (SBP $>160 \mathrm{~mm} \mathrm{Hg}$ and $\mathrm{HR}<60 \mathrm{bpm}$ ) yielded an AUC of 0.65 . Although specificity of this most predictive combination of clinical criteria was high (93.2\%), sensitivity was very low (36.8\%, table 2). Only seven patients had a fixed dilated pupil and fulfilled Cushing's criteria (AUC 0.54 (0.46-0.62)).

Sensitivity analysis of the subgroup of patients with isolated head injuries $(n=92)$ revealed that the combined optimal cutoff values for HR, SBP and pupil size were the same as for the study population as a whole. For code red patients, however, the optimal combination to diagnose a raised ICP was an HR $<70 \mathrm{bpm}$, SBP $<130 \mathrm{~mm} \mathrm{Hg}$ and fixed pupils $>5 \mathrm{~mm}$. Diagnostic accuracy values for both subgroups are presented in table 3.

\section{Clinical criteria in relation to treatment provided}

A total of 43 patients had a fixed dilated pupil $(>5 \mathrm{~mm})$ or a head injury with a Cushing's response (HR $<60 \mathrm{bpm}$ and SBP $>160 \mathrm{~mm} \mathrm{Hg}$ ) anytime in the presence of the HEMS crews. Thirty-two patients received subsequent ICP-lowering treatment with $6 \mathrm{~mL} / \mathrm{kg}$ HS 5\% of whom twenty-seven were found to have a raised ICP. Eleven patients did not receive ICP-lowering treatment, of which five had a raised ICP. Of the 206 patients who did not meet clinical criteria for a raised ICP, 24 were treated with HS, of which 15 were found to have a raised ICP on arrival in hospital, and 182 were not treated with HS of which 40 had a raised ICP (figure 2). Overall, in 190 out of 249 patients (76.3\%) the correct decision was made to administer/withhold HS. Strict adherence to the optimal cut-off points for SBP, HR and pupil size as derived in this study would have resulted in a correct decision in 183/249 (73.5\%) of the patients.

\section{DISCUSSION}

In this study, we demonstrate that clinical signs are a poor predictor of a raised ICP following TBI in the prehospital setting. Treatment decisions based on clinical signs of a raised ICP are correct in around $75 \%$ of the cases. This percentage does not improve when optimal cut-off values (SBP $>160 \mathrm{~mm} \mathrm{Hg}$, $\mathrm{HR}<60 \mathrm{bpm}$ and a fixed pupil $>5 \mathrm{~mm}$ ) are used. Although the specificity of the commonly used combination of clinical criteria (low GCS with fixed dilated pupil(s) and/or Cushing response) is good, sensitivity is poor, and many patients with a raised ICP, signs of significant midline shift, mass effect or (impeding) herniation on arrival in hospital remained undiagnosed.

The low sensitivity of clinical criteria for the prediction of a raised ICP is in line with a previous study investigating the impact of Cushing's sign in the prehospital setting on predicting the need for immediate neurosurgical intervention in trauma patients. ${ }^{12}$ There are several explanations for the low sensitivity as found. First, raised ICP results in pressure gradients between compartments of the brain and a shift of brain structures. ${ }^{23}$ Many of the clinical counterparts of raised ICP are the consequence of such shifts (herniations) rather than the absolute

Table 3 Diagnostic accuracy of clinical criteria to discriminate the presence of a raised ICP in subgroups of patients with isolated head injury $(n=92)$ and code red patients $(n=53)$

\begin{tabular}{|c|c|c|c|c|c|}
\hline & Sensitivity & Specificity & LR+ & LR- & AUC \\
\hline \multicolumn{6}{|l|}{ Isolated TBI } \\
\hline Fixed pupils $>5 \mathrm{~mm}$ or $(\mathrm{HR}<60$ and $\mathrm{SBP}>160)$ & $38.3(24.5-53.6)$ & $95.7(85.2-99.5)$ & $8.8(2.2-35.8]$ & $0.7(0.5-0.8)$ & $0.66(0.54-0.77)$ \\
\hline \multicolumn{6}{|l|}{ Code red } \\
\hline Fixed pupils $>5 \mathrm{~mm}$ or $(\mathrm{HR}<60$ and SBP $>160)$ & $53.8(25.1-80.8)$ & $95.0(83.1-99.4)$ & $10.8(2.6-45.5)$ & $0.5(0.3-0.9)$ & $0.74(0.56-92)$ \\
\hline Fixed pupils $>5 \mathrm{~mm}$ or $(\mathrm{HR}<70$ and $\mathrm{SBP}>130)$ & $61.5(31.6-86.2)$ & $87.5(73.2-95.8)$ & $4.9(2.0-12.4)$ & $0.4(0.2-0.9)$ & $0.75(0.57-0.92)$ \\
\hline
\end{tabular}

AUC, Area Under the Curve; HR, heart rate; LR+, positive likelihood ratio; LR-, negative likelihood ratio; SBP, systolic blood pressure. 


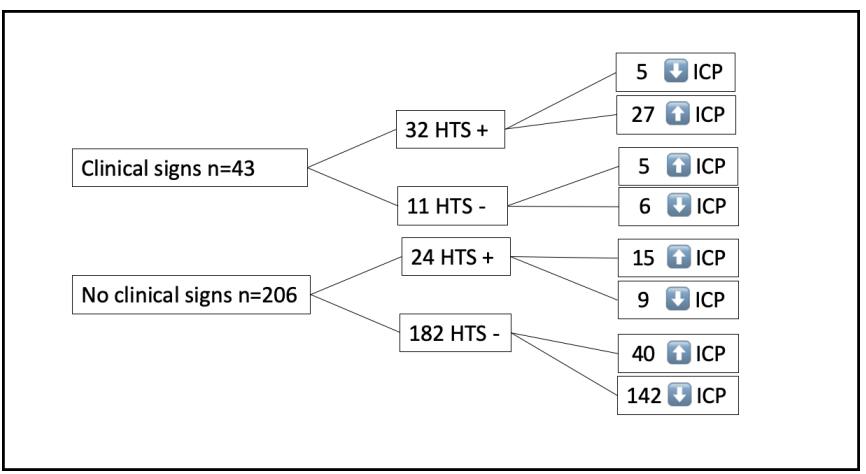

Figure 2 Clinical criteria of increased ICP in relation to treatment provided. HTS, hypertonic saline; ICP, intracranial pressure.

level of ICP. For example, pupillary dilation results from caudal displacement of the diencephalon, whereas a raise in BP and bradycardia result from brainstem distortion. ${ }^{2}$ Both, however, are late signs of a rise in ICP, and significant shift may already be present before these develop. Second, sensitivity of clinical signs may be impaired by the fact that these do not always develop despite significant swelling. This may especially be true in the elderly population where significant transtentorial herniation can occur as a result of a temporal haematoma, without a significant reduction in GCS or development of (other) clinical signs of (impeding) herniation. ${ }^{13}$ Third, overall sensitivity may be impaired due coexisting injuries. Especially in hypovolaemic patients, the traditional Cushing's response may be obscured due to coexisting intravascular volume loss, opposing the effect of the sympathetic surge, thereby contributing to a higher number of false-negative cases. We could not demonstrate this in our study, however, as sensitivity analysis of the subgroup of patients with isolated head injury did not improve diagnostic accuracy significantly. Based on our findings, however, it seems reasonable to use alternative parameters (with a higher HR and lower BP threshold) to define a Cushing's response in patients declared code red, as this improves sensitivity to detect patients with a concomitant rise in ICP. However, due to the relatively small number of code red patients in our cohort, it is hard to recommend specific cut-off values for this cohort. Furthermore, in these patients, maintaining adequate CPP by increasing MAP precedes ICP-lowering treatment to prevent secondary brain injury. Sensitivity may further be affected by spurious reads for vital signs (especially BP), as these are not uncommon in the prehospital situation due to vibrations/movement of the monitor during helicopter or ambulance transport. ${ }^{14}$ Although we aimed to minimise the impact of spurious readings for $\mathrm{HR}$ and $\mathrm{BP}$ on our results by calculating highest $\mathrm{BP}$ and lowest $\mathrm{HR}$ values as averages of 3 consecutive recordings, we cannot exclude this may have contributed to the low sensitivity as found. Finally, as previous studies have shown, ${ }^{15-17}$ considerable variation may be present between clinicians when it comes to judging pupillary diameters.

In our study, 24 out of 206 patients without clinical signs received HS. It is likely that HS was administered off-SOP to these patients based on the trend in their vital signs (eg, progressive bradycardia during transport) rather than absolute numbers. Eleven patients with clinical signs did not receive HS while meeting SOP criteria. Reasons for withholding HS in these patients were not explicitly recorded for most patients, but most likely included improvement of clinical signs with other (standard) measures such as analgesia, neuroprotective ventilation and optimisation of $\mathrm{SpO}_{2}$.

Poor sensitivity of clinical signs to predict ICP may have affected treatment decisions in our study population. Although general ICP-lowering treatment (head up transport, adequate sedation and analgesia, muscle relaxation and neuroprotective ventilation) was provided to all patients after TBI, hyperosmolar therapy may have been withheld in a number of patients with false-negative clinical signs. The implications of this on final outcome of these patients remain unclear: although a (prolonged) raised ICP has been shown to be associated with a worse outcome, a proven survival benefit has never been established for hyperosmolar therapy in patients with TBI. ${ }^{18-20}$ As we found that specificity of clinical signs for a raised ICP is high, overtreatment (with a resulting risk of development of acute heart failure, pulmonary oedema and the possibility of developing central pontine myelinosis $)^{21}$ is less of a concern when treatment guidelines are followed.

Despite the shortcomings of clinical criteria, alternatives are mostly unavailable in the prehospital setting. In hospital, ocular ultrasound with optic nerve sheath diameter measurements has been shown to be a promising tool for detection of an increased ICP. ${ }^{22}$ Alternatively, new portable near-infrared brain scanners may be a promising addition to clinical signs to identify critical pathology at an early stage in the prehospital setting in the near future. ${ }^{2324}$ Until these are available, prehospital clinicians should be aware of the limitations of using clinical signs.

Our study has several limitations. First, due to the retrospective character of the study, selection bias may have influenced our results. We selected patients with blunt head injuries who required prehospital anaesthesia according to the clinical team in attendance, instead of using a cut-off value for GCS, as the latter has been shown to be an unreliable predictor of severe head injury, particularly in elderly trauma patients. ${ }^{14}$ Although unlikely, by doing so, we may have missed patients with severe head injuries in our study population. As a result of our patient selection criteria, breathing pattern (part of Cushing's triad) could not be included in the clinical criteria to detect an increased ICP (as PHEA and subsequent sedation make it an invaluable parameter for continued monitoring during transport). This may have influenced sensitivity of clinical criteria in a negative way. Furthermore, only patients with available outcome data were included; as we do not know if certain groups of patients (either very sick or very well patients) specifically were lost to follow-up, we cannot exclude that missing outcome data/ selection bias may have affected the results. Further, treatment factors such as analgesia, sedation, blood product, crystalloid or HS administration may modify HR and SBP, and thereby influence optimal cut-off points. Outcome was defined by direct ICP measurements CT findings. Although morphological changes in TBI result from an increase in ICP, the relation between intracranial shifts and ICP may vary with age due to brain atrophy. CT findings and ICP measurements may also have been influenced by prior HS administration. Finally, ICP measurements are seldom completed within a short time after arrival to the Emergency Department (ED), and may thereby not reflect values representative of prehospital ICP. However, the number of subjects with direct ICP measurement or craniectomy as an endpoint $(n=12)$ in our study was small, and therefore it is unlikely that this has influenced our results significantly. 


\section{CONCLUSION}

Traditional clinical signs of raised ICP may under triage patients to prehospital treatment with hyperosmolar drugs. Further research should identify more accurate clinical signs or alternative non-invasive diagnostic aids in the prehospital environment.

Twitter Ewoud ter Avest @ewoudteravest

Contributors All authors fulfilled the ICMJE criteria for authorship. ST and RLL conceived the study. ST acquired the data and EtA and ST interpreted the data. EtA drafted the manuscript. All authors revised the manuscript critically and gave final approval to submission of the manuscript.

Funding The authors have not declared a specific grant for this research from any funding agency in the public, commercial or not-for-profit sectors.

Competing interests None declared.

Patient and public involvement Patients and/or the public were not involved in the design, or conduct, or reporting, or dissemination plans of this research.

Patient consent for publication Not required.

Ethics approval This project met National Institute for Healthcare Research (UK) criteria for service evaluation and formal ethical approval was, therefore, not required. The project was approved by the AAKSS Research \& Development Committee. Patients or the public were not involved in the design, or conduct, or reporting, or dissemination plans of our research.

Provenance and peer review Not commissioned; externally peer reviewed.

Data availability statement Deidentified participant data are available upon reasonable request.

Open access This is an open access article distributed in accordance with the Creative Commons Attribution Non Commercial (CC BY-NC 4.0) license, which permits others to distribute, remix, adapt, build upon this work non-commercially, and license their derivative works on different terms, provided the original work is properly cited, appropriate credit is given, any changes made indicated, and the use is non-commercial. See: http://creativecommons.org/licenses/by-nc/4.0/.

ORCID ID

Ewoud ter Avest http://orcid.org/0000-0002-1462-6130

\section{REFERENCES}

1 Dewan MC, Rattani A, Gupta S, et al. Estimating the global incidence of traumatic brain injury. J Neurosurg 2018;1:1-18.

2 Dunn LT. Raised intracranial pressure. J Neurol Neurosurg Psychiatry 2002:73:i23-7.

3 Smith M. Cerebral perfusion pressure. Br J Anaesth 2015;115:488-90.

4 Fodstad H, Kelly PJ, Buchfelder M. History of the cushing reflex. Neurosurgery 2006;59:1132-7.
5 Geeraerts T, Velly L, Abdennour L, et al. Management of severe traumatic brain injury (first 24 hours). Anesth Crit Care Pain Med 2018;37:171-86.

6 Hoogmartens $\mathrm{O}$, Heselmans A, Van de Velde S, et al. Evidence-based prehospital management of severe traumatic brain injury: a comparative analysis of current clinical practice guidelines. Prehosp Emerg Care 2014;18:265-73.

7 Badjatia N, Carney N, Crocco TJ, et al. Guidelines for prehospital management of traumatic brain injury 2nd edition. Prehosp Emerg Care 2008;12 Suppl 1:S1-52.

8 Carney N, Totten AM, O'Reilly C, et al. Guidelines for the management of severe traumatic brain injury, fourth edition. Neurosurgery 2017;80:6-15.

9 Chesnut RM, Temkin N, Carney N, et al. A trial of intracranial-pressure monitoring in traumatic brain injury. N Eng/ J Med 2012;367:2471-81.

10 Lyon RM, Perkins ZB, Chatterjee D, et al. Significant modification of traditional rapid sequence induction improves safety and effectiveness of pre-hospital trauma anaesthesia. Crit Care 2015;19:134.

11 Maas AIR, Stocchetti N, Bullock R. Moderate and severe traumatic brain injury in adults. Lancet Neurol 2008;7:728-41.

12 Yumoto T, Mitsuhashi T, Yamakawa Y, et al. Impact of Cushing's sign in the prehospital setting on predicting the need for immediate neurosurgical intervention in trauma patients: a nationwide retrospective observational study. Scand I Trauma Resusc Emerg Med 2016;24:147.

13 Kehoe A, Rennie S, Smith JE. Glasgow coma scale is unreliable for the prediction of severe head injury in elderly trauma patients. Emerg Med J 2015;32:613-5.

14 McMahon N, Hogg LA, Corfield AR, et al. Comparison of non-invasive and invasive blood pressure in aeromedical care. Anaesthesia 2012;67:1343-7.

15 Kebapcı A, Topçu S. Interrater reliability in pupillary assessment among intensive care nurses. Intensive Crit Care Nurs 2020:58:102801

16 Olson DM, Stutzman S, Saju C, et al. Interrater reliability of pupillary assessments. Neurocrit Care 2016:24:251-7.

17 Clark A, Clarke TNS, Gregson B, et al. Variability in pupil size estimation. Emerg Med J 2006:23:440-1.

18 Cooper DJ, Myles PS, McDermott FT, et al. Prehospital hypertonic saline resuscitation of patients with hypotension and severe traumatic brain injury: a randomized controlled trial. JAMA 2004:291:1350-7.

19 Chen H, Song Z, Dennis JA. Hypertonic saline versus other intracranial pressurelowering agents for people with acute traumatic brain injury. Cochrane Database Syst Rev 2020;1:CD010904.

20 Strandvik GF. Hypertonic saline in critical care: a review of the literature and guidelines for use in hypotensive states and raised intracranial pressure. Anaesthesia 2009:64:990-1003.

21 Himmelseher S, Suarez JL. Hypertonic saline solutions for treatment of intracranial hypertension. Curr Opin Anaesthesiol 2007;20:414-26.

22 Lee SH, Kim HS, Yun SJ. Optic nerve sheath diameter measurement for predicting raised intracranial pressure in adult patients with severe traumatic brain injury: a meta-analysis. J Crit Care 2020;56:182-7.

23 Kim AL. Portable traumatic brain injury detection with near-infrared technology: infrascanner model 2000. Mil Med 2015;180:597-8.

24 Peters J, Van Wageningen B, Hoogerwerf N, et al. Near-Infrared spectroscopy: a promising prehospital tool for management of traumatic brain injury. Prehosp Disaster Med 2017:32:414-8. 